\title{
TEORIA DE LAS INTELIGENCIAS MÚLTIPLES, UNA ALTERNATIVA PARA MEJORAR LAS HABILIDADES, EN LA ASIGNATURA DE QUÍMICA, EN ESTUDIANTES DE EDUCACIÓN MEDIA.
}

\author{
Alvarado Catalina, catacrazy9@hotmail.com \\ Delgado Erika, mar_cielo_aire@hotmail.com \\ Pinzón Paola, paolacindy@hotmail.com \\ Vaca Diana, dianamu9@hotmail.com \\ Villanueva Carolina scarovs128@hotmail.com ${ }^{1}$
}

\section{RESUMEN}

A lo largo de los años, se han dado a conocer diferentes corrientes pedagógicas con la finalidad de mejorar los procesos de enseñanza aprendizaje, en Colombia por mucho tiempo se ha adoptado una corriente tradicional donde el contenido es algo memorístico, mecánico y en realidad muy poco analítico, de modo que se torna aburrido para el educando y genera desinterés por aprender, en consecuencia se pretende implementar un estilo de enseñanza, partiendo de la Teoría de las Inteligencias Múltiples (Howard Gardner, 1983), con el fin de desarrollar las inteligencias en las estudiantes de la IED Liceo Femenino Mercedes Nariño. ya que "La idea fundamental de la teoría es que no existe una sola capacidad mental subyacente, sino una variedad de inteligencias que actúan en combinación y son las que ayudan a explicar cómo las personas adoptan diversos roles dentro de una sociedad2" (Gardner, 2001). Este trabajo se realiza a partir de un portafolio, que contiene actividades que potencializan algunas inteligencias múltiples.

\section{Palabras Clave}

Inteligencia, Inteligencias Múltiples, Portafolio, Habilidades.

\footnotetext{
${ }^{1}$ Estudiantes del Departamento de Química.
} 


\section{JUSTIFICACIÓN}

Partiendo de la psicología cognitiva, que establece que el hombre es precursor activo de la información, y de los estudios realizados por Gardner sobre las inteligencias múltiples, se determina que cada ser humano presenta diferentes actitudes donde se ven involucradas las habilidades y destrezas al realizar una determinada tarea.

De modo que siendo la Química una ciencia de difícil comprensión para el estudiantado, se hace necesario desarrollar algunas de las Inteligencias Múltiples, a través de actividades complementarias en el aula de clase por medio de un portafolio donde el docente y las estudiantes incorporen muestras significativas del trabajo realizado durante el desarrollo de las clases, y así despertar el interés, el gusto por la Química.

\section{REFERENTES CONCEPTUALES}

A partir de la Teoría de Inteligencias Múltiples (Gardner, 1983), se conoce que existen diversas inteligencias y que por esta razón las habilidades de los estudiantes varían según su desarrollo, sin embargo cuando se analizan los programas de enseñanza que se imparten, los profesores de cierta manera "obligan" a los alumnos a concentrarse en el predominio de las inteligencias lingüística y matemática, dando mínima importancia a las otras posibilidades del conocimiento, aquí el por qué muchos alumnos que no se destacan en el dominio de las inteligencias académicas tradicionales, han disminuido sus habilidades hasta llegar a pensar que han fracasado, cuando en realidad los profesores son los que están suprimiendo sus talentos.
*Teoría de las Inteligencias Múltiples

La Teoría de las Inteligencias Múltiples, propuesta por el psicólogo norteamericano Howard Gardner, se fundamenta en el hecho de que cada persona tiene por lo menos ocho- inteligencias u ocho habilidades cognoscitivas, dicha teoría expuesta específicamente en su libro Frames of Mind: TheTheory of Multiplelntelligences (1983), en el cual las define como "la capacidad de resolver problemas y/o elaborar productos habituales que son importantes en un ámbito cultural o en una comunidad determinada." (Gardner, 2001); Howard Gardner y su equipo de la universidad de Harvard han identificado ocho tipos distintos de inteligencias:

-Inteligencia verbal-lingüística: Capacidad de emplear palabras eficazmente, bien sea en forma oral o escrita, comprende la habilidad de manipular la sintaxis, la fonética y la semántica del lenguaje.

-Inteligencia lógico-matemática: Capacidad para comprender relaciones, patrones lógicos, enunciados y propuestas, funciones y otras abstracciones afines.

-Inteligencia visual-espacial: Habilidad de percibir acertadamente el mundo visual y espacial, transformando esas percepciones en conceptos, implica ser sensible al color, la forma, las figuras, el espacio y la relación que existe entre estos elementos.

Inteligencia Corporal-cinestésica: Habilidad de utilizar el cuerpo para expresar emociones, para realizar juegos o para idear nuevos inventos, ésta comprende habilidades físicas específicas como la coordinación motora y el equilibrio entre otras. 
-Inteligencia interpersonal-social: Se relaciona con la facultad de percibir y distinguir los estados de ánimo, intenciones, motivos, deseos y sentimientos de otras personas a través de sus expresiones faciales, su voz y gestos.

-Inteligencia intrapersonal - introspectiva: Implica el conocimiento propio y la habilidad de actuar conforme a ello, esta conlleva a tener una imagen acertada de sí mismo.

-Inteligencia musical: Capacidad para percibir, distinguir, transformar y expresar sonidos y formas musicales, esta inteligencia comprende la facultad de discernir los sonidos del ambiente.

\section{Aplicaciones de la Teoría de la IM}

De acuerdo con la propuesta de Gardner, se han venido realizando diferentes proyectos para generar diversas propuestas pedagógicas en pro del mejoramiento de la calidad de la educación, desde esta perspectiva, algunos países han realizado varias investigaciones, las cuales son utilizadas en una reestructuración en la práctica pedagógica y una alternativa para los futuros estudiantes que, día a día, manifiestan diversidad en sus capacidades. Dentro de los trabajos realizados se encuentran el Proyecto Programon International Financial Systems (PIFS), en este proyecto se enseña al estudiante a aprovechar al máximo sus habilidades meta-cognitivas, controlar su aprendizaje y favorecer sus interacciones con los demás.

Algunos componentes para desarrollar este proyecto son: identificación de problemas, elección de una estrategia para ordenar los pasos para la solución de los problemas, asignación de los recursos, control del proceso de solución y evaluación de los resultados, desde esta perspectiva se plantea la idea de un portafolio donde el docente y los alumnos incorporen muestras significativas del trabajo realizado durante el ciclo escolar. El concepto de portafolios se refiere al conjunto de evaluaciones y actividades cuyo objetivo es valorar las competencias de los estudiantes dentro del contexto de aprendizaje, este favorece la creación de un entorno donde el estudiante y profesor interactúan dando un enfoque a una evaluación personalizada.

\section{PROBLEMA}

La Química es un área del conocimiento que conlleva muchas veces a un aprendizaje memorístico y mecánico, como lo es el caso de la Nomenclatura Inorgánica y Reacciones Químicas, que se fundamentan en normas específicas, por lo que se ha evidenciado un desinterés frente al área por parte de las estudiantes, lo que se refleja en la evaluación, por lo tanto, se pretende desarrollar actividades que pongan de manifiesto algunas de las Inteligencias Múltiples, ya que éstas ofrecen una alternativa para que se motiven en cuanto al aprendizaje.

\section{OBJETIVOS}

Los objetivos propuestos para este trabajo son

\section{* General}

Promover en las estudiantes el desarrollo de algunas de las Inteligencias Múltiples, utilizando un portafolio que contiene actividades que despierten el interés de las estudiantes por la Nomenclatura de compuestos inorgánicos y Reacciones Químicas.

\section{${ }^{*}$ Específicos}

1. Identificar las inteligencias múltiples en las estudiantes. 
2. Diseñar actividades para el portafolio orientadas al desarrollo de algunas inteligencias múltiples

3. Establecer el proceso que lleva cada estudiante durante el desarrollo de la asignatura.

4. Mejorar el rendimiento académico de las estudiantes, en los temas trabajados en clase, por medio de las actividades desarrolladas en el portafolio.

\section{METODOLOGÍA}

La realización del proyecto consta de cuatro fases:

- Fase diagnóstico. Se realiza una caracterización de las inteligencias múltiples de las estudiantes a través de un Test de Inteligencias Múltiples (Armstrong, 1995) (Anexo 1), el cual consta de 35 afirmaciones que buscan identificar el nivel de desarrollo de las inteligencias en las estudiantes de grado noveno (2010-II) y décimo (2011-I) del Liceo Femenino Mercedes Nariño.

- $\quad$ Fase de diseño. Esta etapa parte de los resultados obtenidos del Test de Inteligencias Múltiples; se elaboraron actividades para el tema de Nomenclatura y Reacción Química, con el fin de desarrollar las inteligencias: lingüística, visualespacial y lógico-matemática, recopiladas en un portafolio que consta de 16 actividades enfocadas a desarrollar el lenguaje científico de la Nomenclatura Inorgánica y la comprensión lectora, desde la perspectiva lingüística. En cuanto a la lógica- matemática, contribuye a la comprensión de formación de compuestos, clasificación de grupos funcionales y balanceo de ecuaciones de acuerdo con la temática trabajada en clase, y por último, la visual- espacial es promovida a través de diagramas que diferencian la posición de los elementos en la tabla periódica. En plataforma Actiweb, aparecen las actividades diseñadas y subidas a la página por las autoras. www.actiweb.es/quimica.

- $\quad$ Fase de Aplicación. Se aplica y se desarrolla cada una de las actividades propuestas en la fase anterior con las estudiantes de grado decimo.

- $\quad$ Fase de evaluación. Para verificar si la propuesta tuvo alguna incidencia en el desarrollo de las diferentes inteligencias en las estudiantes de grado décimo, se aplicó nuevamente el test de las inteligencias Múltiples de Gardner (Anexo 1), y se evaluaron los resultados obtenidos con el portafolio, empleando la matriz de valoración elaborada por las autoras del proyecto (Anexo 2).

\section{RESULTADOS Y ANÁLISIS}

Para evaluar el desarrollo de las diferentes inteligencias en las estudiantes, se empleó el test de Inteligencias Múltiples desarrollado y validado por Gardner; este se aplicó como pretest y postest para verificar si el trabajo con el portafolio contribuye al desarrollo de las inteligencias. 
Los resultados obtenidos en la aplicación inicial y final del Test IM arrojaron los siguientes resultados:

Gráfica 1: Inteligencias múltiples

I.M.

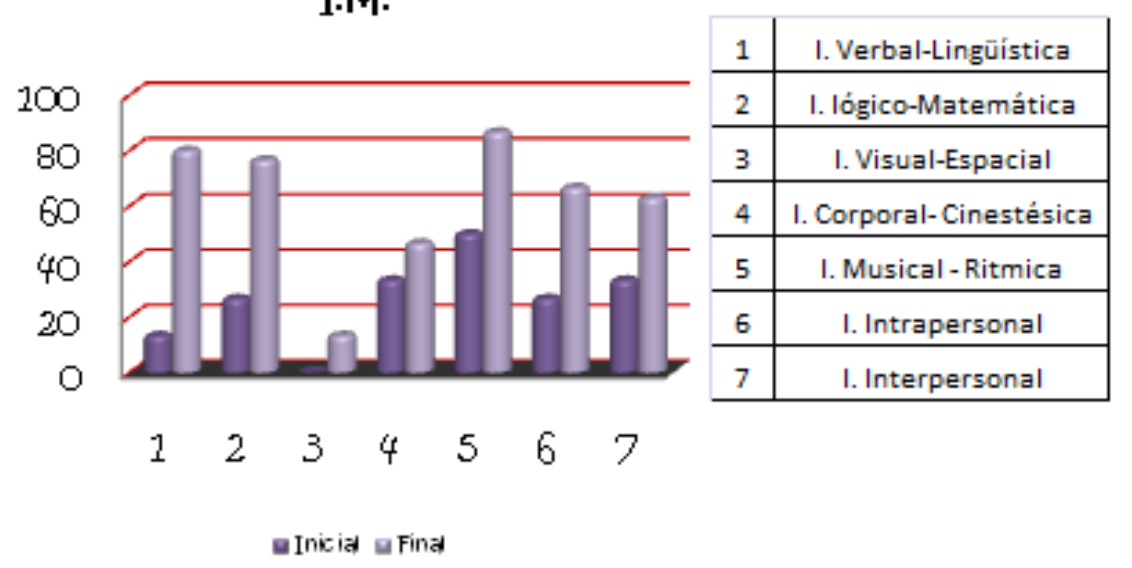

De acuerdo con la anterior gráfica, las inteligencias más desarrolladas en la primera aplicación del test IM son la musical-rítmica y corporal-cinestésica, ya que éstas se desarrollan a la par con la cultura, es decir, el área de educación física, las danzas, la música, han estado siempre inmersas en el currículo a nivel de educación preescolar, básica y media como área fundamental, de igual forma el contacto social con la comunidad hace que éstas inteligencias sobresalgan mucho más; las inteligencias que menos han desarrollado son: visual-espacial, intrapersonal, interpersonal, verbal - lingüística y lógico- matemática (gráfica 1), según la información obtenida.

Para verificar la viabilidad de la propuesta, se aplica por segunda vez el test de IM, en el cual se evidencia el desarrollo de las inteligencias: lógicomatemática, lingüística, interpersonal y visual espacial, además de las ya desarrolladas, como la Inteligencia corporal - cinestésica, Musical e Intrapersonal, aunque no se diseñaron actividades para desarrollar dichas inteligencias, éstas se mantienen, ya que van entrelazadas unas con otras y hacen parte de su diario vivir.

Según el desarrollo de las actividades propuestas en el portafolio, se obtuvieron los siguientes resultados (Gráfica $\mathrm{N}^{\circ} 2$ ), de acuerdo con los criterios de la matriz de evaluación que se realizó para el análisis de las mismas (Ver anexo 2). 


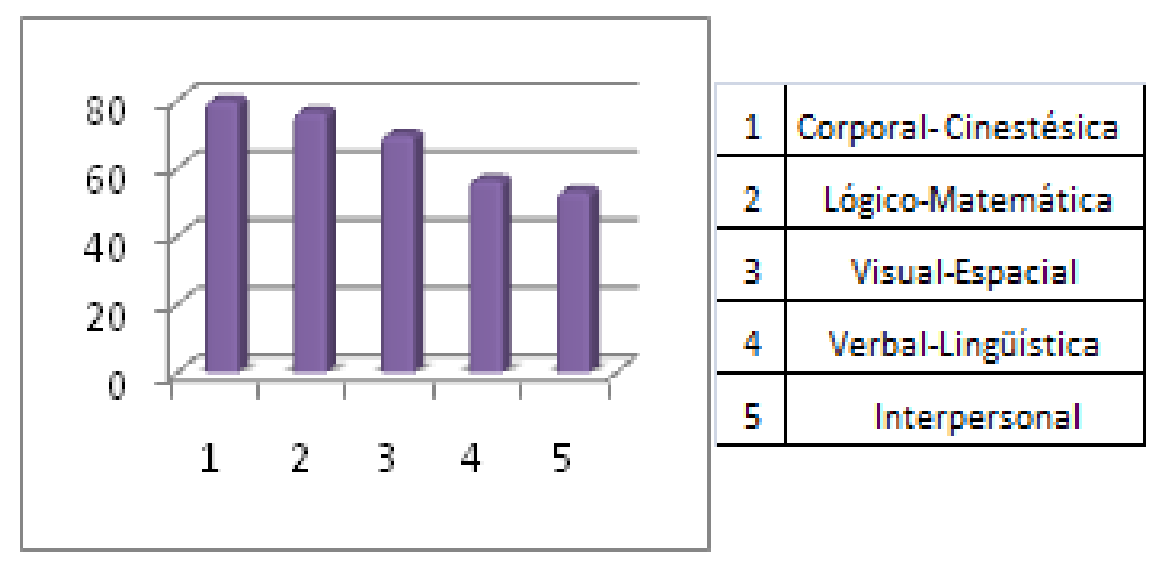

Gráfica 2: Inteligencias múltiples desarrolladas en el portafolio

Con base en los objetivos de las actividades y los resultados anteriores, se infiere que el portafolio contribuye al desarrollo de las inteligencias lógicomatemática, lingüística, interpersonal, visual - espacial, corporal - cinestésica, e interpersonal, esto se evidencia a través de una participación activa de las estudiantes en las clases, ya que el portafolio generó un interés provocando un cambio de actitud frente a la asignatura.

Por último se realiza una actividad grupal, donde las estudiantes dan a conocer de forma creativa un producto cotidiano a través de un video; la finalidad de este fue abarcar en su mayoría las inteligencias que se querían potencializar, desde el inicio del proyecto. Al analizar los resultados de la actividad se encontró que las inteligencias corporal- cinestésica, interpersonal y lingüística se han desarrollado, teniendo en cuenta los criterios de análisis.

Finalmente los resultados obtenidos en la aplicación de la propuesta, indican que las actividades del portafolio y la actividad grupal contribuyen al desarrollo de las inteligencias, debido a que el trabajo realizado dentro y fuera de las clases favorece el desarrollo de estas, además del cambio hacia una actitud favorable y un mejor desempeño frente a la asignatura.

\section{CONCLUSIONES}

- Las inteligencias múltiples no se pueden desarrollar a corto plazo, estas se deben abordar mediante actividades continuas que fortalezcan una determinada habilidad y por consiguiente la inteligencia. La evolución de cada una de éstas, depende de la voluntad y del trabajo que realiza la estudiante para

fortalecer sus inteligencias, donde el acompañamiento del docente como guía es fundamental.

- El portafolio sirve como un registro sistemático y organizado del proceso de aprendizaje, centrándose en el trabajo de la estudiante y su reflexión sobre el mismo; mediante un esfuerzo cooperativo entre estudiante - docente en formación. El portafolio como técnica de aprendizaje, resulta significativo en el 
desarrollo de clases y obtención de resultados académicos, ya que mejora la actitud de las estudiantes frente al trabajo de aula, aumentando su comprensión y aprehensión de los conceptos estructurantes de la temática, aunque esto significa que el trabajo del docente sea más arduo al momento de planear las actividades y la dirección de las clases de forma más personalizada.
- De acuerdo con currículo que se trabaja en el colegio (2011I), muchas de las áreas desarrollan implícitamente algunas inteligencias múltiples pero no se tiene un enfoque de su potencializacion y por ello su poco desarrollo.

\section{Bibliografía}

- $\quad$ Armstrong, T. (1995) Inteligencias múltiples en el salón de clases.

Gardner Howard, (2001), Estructuras de la mente. La teoría de las múltiples inteligencias, F.C.E., México

- Gomis, N. (2007). Tesis doctoral: Evaluación de las Inteligencias Múltiples en el contexto educativo a través de expertos, maestros y padres. Universidad de Alicante. Departamento de Psicología Evolutiva y Didáctica. Recuperado el 3 de agosto 2010 de la World Wide Web: http:// www.cervantesvirtual.
- $\quad \mathrm{com} / \mathrm{servlet/SirveObras/5681628}$ 432903611061057 /031975.pdf

- Lozano, E. (2008). Inteligencias múltiples en el aula. Disertación doctoral publicada. Universidad de Murcia, facultad de Educación departamento de Psicología Evolutiva y de la Educación. Murcia.

- National Education Association. Recuperado el 14 de octubre 2010 de la World Wide Web: http:// www.nea.org/ 


\section{ANEXOS}

\section{TEST DE LAS INTELIGENCIAS MÚLTIPLES (Armstrong, 1995)}

Este test te ayudará a que puedas conocerte mejor $\mathrm{y}$, también, a que puedas identificar las áreas más sobresalientes de tu inteligencia. Por favor, responde con mucha honestidad y sinceridad. Tenga en cuenta la siguiente instrucción:

a.- Si crees que refleja una característica tuya y te parece que la afirmación es verdadera, escribe "V".

b.- Si crees que no refleja una característica tuya y te parece que la afirmación es falsa, escribe "F".

c.- Si estás dudoso porque a veces es verdadera y a veces falsa no escribas nada y déjala en blanco.

\begin{tabular}{|c|c|}
\hline 1 & Prefiero hacer un mapa que explicarle a alguien como tiene que llegar a un lugar determinado \\
\hline 2 & Si estoy enojado o contento generalmente sé la razón exacta de por qué es así \\
\hline 3 & Sé tocar, o antes sabía, un instrumento musical. \\
\hline 4 & Asocio la música con mis estados de ánimo \\
\hline 5 & Puedo sumar o multiplicar mentalmente con mucha rapidez \\
\hline 6 & $\begin{array}{l}\text { Puedo ayudar a un amigo (a) a manejar y controlar sus sentimientos, porque yo lo pude hacer antes } \\
\text { en relación a sentimientos parecidos }\end{array}$ \\
\hline 7 & Me gusta trabajar con calculadora y computadoras \\
\hline 8 & Aprendo rápidamente a bailar un baile nuevo \\
\hline 9 & No me es difícil decir lo que pienso durante una discusión o debate. \\
\hline 10 & ¿Disfruto de una buena charla, prédica o sermón? \\
\hline 11 & Siempre distingo el Norte del Sur, esté donde esté. \\
\hline 12 & Me gusta reunir grupos de personas en una fiesta o evento especial. \\
\hline 13 & Realmente la vida me parece vacía sin música \\
\hline 14 & Siempre entiendo los gráficos que vienen en las instrucciones de equipos o instrumentos. \\
\hline 15 & Me gusta resolver puzles y entretenerme con juegos electrónicos \\
\hline
\end{tabular}




\section{Continuación}

\section{TEST DE LAS INTELIGENCIAS MÚLTIPLES (Armstrong, 1995)}

\begin{tabular}{|c|c|}
\hline 16 & Me fue fácil aprender a andar en bicicleta o patines \\
\hline 17 & Me enojo cuando escucho una discusión o una afirmación que me parece ilógica o absurda. \\
\hline 18 & Soy capaz de convencer a otros que sigan mis planes o ideas. \\
\hline 19 & Tengo buen sentido del equilibrio y de coordinación \\
\hline 20 & $\begin{array}{l}\text { A menudo puedo captar relaciones entre números con mayor rapidez y facilidad que algunos de mis } \\
\text { compañeros }\end{array}$ \\
\hline 21 & Me gusta construir modelos, maquetas o hacer esculturas \\
\hline 22 & Soy bueno para encontrar el significado preciso de las palabras. \\
\hline 23 & Puedo mirar un objeto de una manera y con la misma facilidad verlo dado vuelta o al revés. \\
\hline 24 & $\begin{array}{l}\text { Con frecuencia establezco la relación que puede haber entre una música o canción y algo que haya } \\
\text { ocurrido en mi vida. }\end{array}$ \\
\hline 25 & Me gusta trabajar con números y figuras \\
\hline 26 & Me gusta sentarme muy callado y pensar, reflexionar sobre mis sentimientos más íntimos. \\
\hline 27 & Solamente con mirar las formas de las construcciones y estructuras me siento a gusto. \\
\hline 28 & Cuando estoy en la ducha, o cuando estoy solo me gusta tararear, cantar o silbar. \\
\hline 29 & Soy bueno para el atletismo \\
\hline 30 & Me gusta escribir cartas largas a mis amigos. \\
\hline 31 & Generalmente me doy cuenta de la expresión o gestos que tengo en la cara. \\
\hline 32 & Muchas veces me doy cuenta de las expresiones o gestos en la cara de las otras personas. \\
\hline 33 & Reconozco mis estados de ánimo, no me cuesta identificarlos. \\
\hline 34 & Me doy cuenta de los estados de ánimo de las personas con quienes me encuentro \\
\hline 35 & Me doy cuenta bastante bien de lo que los otros piensan de mí. \\
\hline
\end{tabular}




\section{Criterios utilizados para la identificación de las Inteligencias Múltiples}

\begin{tabular}{|c|c|}
\hline INTELIGENCIA & PREGUNTAS NO. \\
\hline Inteligencia verbal/Lingüística & $9,10,17,22,30$ \\
\hline Inteligencia Lógica/Matemática & $5,7,15,20,25$ \\
\hline Inteligencia Visual/Espacial & $1,11,14,23,27$ \\
\hline Inteligencia Corporal/Cinestésica & $8,16,19,21,29$ \\
\hline Inteligencia Musical/Rítmica & $3,4,13,24,28$ \\
\hline Inteligencia Intrapersonal & $2,6,26,31,33$ \\
\hline Inteligencia Interpersonal & $12,18,32,34,35$ \\
\hline
\end{tabular}

\section{Matriz de criterios para evaluar las actividades del portafolio}

\begin{tabular}{|c|c|c|}
\hline ACTIVIDAD & INTELIGENCIA & CRITERIOS \\
\hline \multirow[t]{2}{*}{$\begin{array}{l}\text { 1. Aprendiendo } \\
\text { Conceptos }\end{array}$} & Lingüística & $\begin{array}{ll}\diamond & \text { Consulta en libros, los conceptos para comprenderlos. } \\
\diamond & \text { Hace uso de la ortografía para escribir las definiciones. } \\
\diamond & \text { Comprende el objetivo de la actividad. } \\
\diamond & \text { Tiene coherencia para redactar el significado del concepto. }\end{array}$ \\
\hline & Lógica & $\begin{array}{l}\diamond \text { Comprende la estructura de la actividad. } \\
\diamond \text { Encuentra los términos expuestos en el pasatiempo. }\end{array}$ \\
\hline
\end{tabular}




\section{Continuación}

\section{Matriz de criterios para evaluar las actividades del portafolio}

\begin{tabular}{|c|c|c|c|}
\hline \multirow[b]{3}{*}{ 2. Laberinto } & Espacial & $\diamond$ & $\begin{array}{l}\text { Usa otras dimensiones para moverse en el laberinto. } \\
\text { Visualiza con precisión el camino verdadero }\end{array}$ \\
\hline & Lingüística & $\diamond$ & $\begin{array}{l}\text { Escribe correctamente el nombre de cada compuesto. } \\
\text { Tiene comprensión lectora. }\end{array}$ \\
\hline & Lógica & $\diamond$ & $\begin{array}{l}\text { Comprende instrucciones. } \\
\text { Analiza la situación } \\
\text { Hace uso del razonamiento para el desarrollo de la actividad. } \\
\text { Resuelve favorablemente la actividad } \\
\text { Analiza las situaciones de causa y efecto. } \\
\text { Resuelve asertivamente el problema de lógica }\end{array}$ \\
\hline \multirow{3}{*}{$\begin{array}{l}\text { 3.Completando } \\
\text { ecuaciones }\end{array}$} & Lingüística & $\diamond$ & $\begin{array}{l}\text { Entiende lo que se le pide. } \\
\text { Escribe adecuadamente los símbolos de los elementos. }\end{array}$ \\
\hline & Lógica & $\diamond$ & $\begin{array}{l}\text { Completa la ecuación planteada. } \\
\text { Escribe bien los subíndices. } \\
\text { Comprende la síntesis de óxidos. }\end{array}$ \\
\hline & Matemática & $\diamond$ & Maneja bien el concepto de balanceo de ecuaciones. \\
\hline $\begin{array}{l}4 \text { ¿Cómo funcionan } \\
\text { los antiácidos? }\end{array}$ & Lingüística & $\diamond$ & $\begin{array}{l}\text { Analiza la información e identifica los compuestos. } \\
\text { Utiliza sus palabras para mostrar la importancia de los antiácidos. }\end{array}$ \\
\hline 5. Caries dental & Lingüística & $\diamond$ & Analiza la información e identifica los compuestos \\
\hline \multirow[b]{2}{*}{$\begin{array}{l}\text { 6. Explorando tu } \\
\text { entorno }\end{array}$} & Lingüística & $\diamond$ & Investiga otros usos de los compuestos \\
\hline & Lógica & $\diamond$ & $\begin{array}{l}\text { Lee e interpreta el procedimiento } \\
\text { Contribuye a la resolución de una incógnita de su entorno }\end{array}$ \\
\hline \multirow{2}{*}{ 7. Secretos mágicos } & Lingüística & $\diamond$ & $\begin{array}{l}\text { Utiliza su conocimiento científico para expresar el fenómeno. } \\
\text { Describe de manera adecuada la reacción química que se da en el } \\
\text { proceso experimental }\end{array}$ \\
\hline & Lógica & $\diamond$ & $\begin{array}{l}\text { Realiza el procedimiento de modo correcto, obteniendo el objeti- } \\
\text { vo experimental. }\end{array}$ \\
\hline $\begin{array}{l}\text { 8. Ácidos binarios y } \\
\text { ternarios }\end{array}$ & Lingüística & $\checkmark$ & $\begin{array}{l}\text { Identifica los diferentes tipos de ácidos. } \\
\text { Emplea adecuadamente el lenguaje químico, logrando reconocer } \\
\text { el nombre de diferentes compuestos químicos. } \\
\text { Realiza asertivamente las ecuaciones de formación de ácidos. }\end{array}$ \\
\hline
\end{tabular}




\section{Matriz de criterios para evaluar las actividades del portafolio}

\begin{tabular}{|c|c|c|c|}
\hline \multirow[t]{2}{*}{ 9. Formando sales } & Lingüística & $\diamond$ & $\begin{array}{l}\text { Emplea adecuadamente la información para la for- } \\
\text { mación de distintas sales. } \\
\text { Entiende el concepto de sal. }\end{array}$ \\
\hline & Lógica & $\diamond$ & $\begin{array}{l}\text { Comprende e interpreta el enunciado correctamen- } \\
\text { te. }\end{array}$ \\
\hline \multirow{3}{*}{$\begin{array}{l}\text { 10. Formando } \\
\text { compuestos }\end{array}$} & Espacial & $\diamond$ & $\begin{array}{l}\text { Comprende e interpreta el enunciado correctamen- } \\
\text { te. }\end{array}$ \\
\hline & Lingüística & $\diamond$ & $\begin{array}{l}\text { Identifica los símbolos según corresponde para } \\
\text { nombrar los compuestos. } \\
\text { Analiza y escribe los nombres según el numero de } \\
\text { oxidación. }\end{array}$ \\
\hline & Lógica & $\diamond$ & $\begin{array}{l}\text { Comprende relaciones para formar compuesto or- } \\
\text { denadamente. } \\
\text { Agrupa por categorías satisfactoriamente }\end{array}$ \\
\hline \multirow{2}{*}{$\begin{array}{l}\text { 11. Clasificando y } \\
\text { nombrando compuestos }\end{array}$} & Lingüística & $\diamond$ & $\begin{array}{l}\text { Identifica los símbolos según corresponde para } \\
\text { nombrar los compuestos. } \\
\text { Relaciona el compuesto con los números de oxida- } \\
\text { ción y así darle el nombre. }\end{array}$ \\
\hline & Lógica & $\diamond$ & $\begin{array}{l}\text { Comprende relaciones para identificar las funciones } \\
\text { químicas. } \\
\text { Clasifica por categorías satisfactoriamente. }\end{array}$ \\
\hline $\begin{array}{l}\text { 12. Determinando } \\
\text { compuestos }\end{array}$ & Lógica - matemática & $\diamond$ & $\begin{array}{l}\text { Emplea los números correctamente. } \\
\text { Analiza información, busca orden y determina el } \\
\text { número de oxidación correcto. } \\
\text { Realiza cálculos correctamente }\end{array}$ \\
\hline \multirow{3}{*}{$\begin{array}{l}\text { 13. Nombrando } \\
\text { compuestos }\end{array}$} & Lingüística & $\diamond$ & $\begin{array}{l}\text { Comprende lo que se le pregunta. } \\
\text { Escribe de manera adecuada los diferentes com- } \\
\text { puestos. } \\
\text { Desarrolla de manera adecuad el lenguaje químico. }\end{array}$ \\
\hline & Lógica - matemática & $\diamond$ & $\begin{array}{l}\text { Reconoce y forma compuestos químicos de manera } \\
\text { lógica. } \\
\text { Identifica las funciones químicas. } \\
\text { Utiliza adecuadamente los números de oxidación }\end{array}$ \\
\hline & Visual -Espacial & $\diamond$ & $\begin{array}{l}\text { Reconoce los elementos metales de los no metales. } \\
\text { Hace uso de diagramas para realizar compuestos. }\end{array}$ \\
\hline
\end{tabular}


Continuación

3. Matriz de criterios para evaluar las actividades del portafolio

\begin{tabular}{|c|c|c|c|}
\hline \multirow{3}{*}{ 14. Reconocimiento } & Lingüística & $\diamond$ & Responde de acuerdo a lo que se le pregunta \\
\hline & Lógica -matemática & $\diamond$ & $\begin{array}{l}\text { Desarrolla de forma lógica la actividad. } \\
\text { Identifica y escribe los grupos funcionales de forma adecua- } \\
\text { da. } \\
\text { Reconoce que las funciones químicas presentan estructuras } \\
\text { definidas. }\end{array}$ \\
\hline & Visual espacial & $\diamond$ & $\begin{array}{l}\text { Hace uso de diagramas para reconocer los metales de los no } \\
\text { metales. }\end{array}$ \\
\hline \multirow[b]{2}{*}{ 15. Sudoku químico } & Lingüística & $\diamond$ & $\begin{array}{l}\text { Comprende instrucciones escritas y orales que orientas la } \\
\text { actividad. }\end{array}$ \\
\hline & Lógica & $\diamond$ & $\begin{array}{l}\text { Comprende instrucciones. } \\
\text { Analiza las instrucciones y los resultados que obtiene. } \\
\text { Hace uso del razonamiento para un desarrollar favorable- } \\
\text { mente la actividad. } \\
\text { Resuelve el problema de lógica. } \\
\text { Comprende la relación entre cada fórmula que se le pide. } \\
\text { Desarrolla análisis de causa y efecto. } \\
\text { Comprende el orden de la actividad trabajada }\end{array}$ \\
\hline \multirow{3}{*}{ 16. Envídiate } & Corporal & $\diamond$ & $\begin{array}{l}\text { Utiliza el cuerpo para expresar sus ideas. } \\
\text { Emplea los espacios para desarrollar el tema de forma ade- } \\
\text { cuada. } \\
\text { Hace uso de los elementos para dar a conocer el tema }\end{array}$ \\
\hline & Interpersonal & $\diamond$ & $\begin{array}{l}\text { Se evidencia el trabajo en equipo. } \\
\text { Participa activamente en el video. } \\
\text { Demuestra dedicación en la realización del video. }\end{array}$ \\
\hline & Verbal lingüística & $\diamond$ & $\begin{array}{l}\text { Nombra correctamente el compuesto. } \\
\text { Utiliza de manera adecuada el lenguaje químico. } \\
\text { Expresa de manera clara sus ideas. }\end{array}$ \\
\hline
\end{tabular}




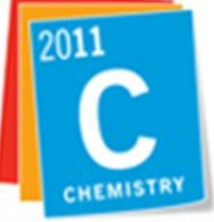

International Year of CHEMISTRY 2011 